\title{
Compositional Safety Refutation Techniques
}

\author{
Kumar Madhukar ${ }^{1,3}$, Peter Schrammel ${ }^{2}$, and Mandayam Srivas ${ }^{3}$ \\ 1 TCS Research, Pune, India \\ 2 University of Sussex, School of Engineering and Informatics, Brighton, UK \\ ${ }^{3}$ Chennai Mathematical Institute, Chennai, India
}

\begin{abstract}
One of the most successful techniques for refuting safety properties is to find counterexamples by bounded model checking. However, for large programs, bounded model checking instances often exceed the limits of resources available. Generating such counterexamples in a modular way could speed up refutation, but it is challenging because of the inherently non-compositional nature of these counterexamples. We start from the monolithic safety verification problem and present a stepby-step derivation of the compositional safety refutation problem. We give three algorithms that solve this problem, discuss their properties with respect to efficiency and completeness, and evaluate them experimentally.
\end{abstract}

\section{Introduction}

Divide-and-conquer approaches are considered to be the blue print solution to scale algorithms to large problems. Compositionality of proofs is the enabler of a map-reduce approach to verification. Compositional verification approaches based on contracts and summaries have been shown to tremendously increase scalability and productivity in real-world formal verification [2|12|19|27].

But what about refutation? Unlike verification, refutation algorithms are usually based on finding a violating execution trace, which seems to be inherently non-compositional. Consequently, the study of the compositional refutation problem is an under-explored area of research. Yet, solutions to this problem have significant impact on other research problems. As a motivation, we give here two algorithmic approaches in verification and testing that will be enabled by efficient compositional refutation algorithms:

- Property-guided abstraction refinement algorithms like CEGAR [6] need to decide whether counterexamples that are found in the abstraction are spurious or true counterexamples. The lack of compositional refutation techniques forces these algorithms to operate in a monolithic manner and is therefore an obstacle to scaling them to large programs.

- Automated test generation techniques based on Bounded Model Checking are successfully used in various industries to generate unit tests (e.g. [25]). However, they do not sufficiently scale to accomplish the task of generating integration tests. Compositional refutation techniques achieve exactly this goal: they efficiently produce refutations (from which test vectors can be 
derived) on unit (module) level and enable their composition in order to obtain system level refutations, i.e. integration tests.

This paper is a first step in this direction and lays the base for a more systematic study of the problem domain.

Contributions. We summarise the contributions of the paper as follows:

- In order to place the problem in a wider context, we give an informal overview on how completeness relates to problem decomposition in safety refutation and verification (Section 3).

- We formalise the safety refutation problem in horizontal decompositions, e.g. procedure-modular decompositions, and characterise the compositional completeness guarantees of various algorithmic approaches (Section 4).

- We describe three refutation approaches with different degrees of completeness (Section 5) and give experimental results on $\mathrm{C}$ benchmarks, comparing their completeness and efficiency (Section 6).

\section{Preliminaries}

Program model and notation. We assume that programs are given in terms of acyclid 4 call graphs, where individual procedures $f$ are given in terms of deterministic, symbolic input/output transition systems. $F$ is the set of all procedures in the program. Since the handling of loops is orthogonal to the compositional aspect, we consider only loop-free procedures (respectively bounded unwindings of loops) in this paper. Thus, we simply denote the input/output relation of a procedure $f$ as $T_{f}\left(\boldsymbol{x}^{\text {in }}, \boldsymbol{x}^{\text {out }}\right)$. Inputs $\boldsymbol{x}^{\text {in }}$ are procedure parameters, global variables, and memory objects that are read by $f$. Outputs $\boldsymbol{x}^{\text {out }}$ are return values, and potential side effects such as global variables and memory objects written by $f$. Boolean guard variables $(g)$ are used to model the control flow. Non-deterministic choices are encoded by additional input variables.

The relations $T_{f}$ are given as first-order logic formulae over bitvectors and arrays, resulting from the logical encoding of the program semantics. Fig. 1 gives an example of the encoding of a program into such formulae using the loop-free notation. The inputs $\boldsymbol{x}^{\text {in }}$ of foo are $\left(y, g_{6}\right)$ and the outputs $\boldsymbol{x}^{\text {out }}$ consist of $\left(r, g_{7}\right)$ where $r$ is the return value. In addition to the inputs and outputs we need boolean guard variables $g^{\text {in }}, g^{\text {out }}$ (here $g_{6}, g_{7}$ ) that are true if the entry and, respectively, exit of the procedure can be reached. They are handled like input/output parameters and have their actual counterparts in the guard variables in the caller (here, e.g. $g_{1}, g_{2}$ for the call $f_{0 o}$ in main). Note that we consider exit in a procedure is not reachable, i.e., $\neg g^{\text {out }}$, if either the program is non-terminating or an assertion in a procedure is violated. Hence, the exit guard condition in the definition of a transition function includes assertion checks as in $T_{b a r}$. We use a single static assignment (SSA) encoding, which gives a fresh name to each update of a variable if it is modified multiple times, such as for example in main.

\footnotetext{
${ }^{4}$ We consider non-recursive programs with multiple procedures (cf. model in [5]).
} 
that call site. We provide formal definitions for summaries and calling contexts below (invariants are not needed in this paper).

Definition 1. For a procedure given by $T_{f}$ we define:

- A summary is a predicate Sum $_{f}$ such that:

$$
\forall X_{f}: T_{f}\left(\boldsymbol{x}^{\text {in }}, \boldsymbol{x}^{\text {out }}\right) \Longrightarrow \operatorname{Sum}_{f}\left(\boldsymbol{x}^{\text {in }}, \boldsymbol{x}^{\text {out }}\right)
$$

- The calling context for a procedure call $h$ at call site $i$ in the given procedure is a predicate CallCtx $x_{i}$ such that

$$
\forall X_{f}: T_{f}\left(\boldsymbol{x}^{\text {in }}, \boldsymbol{x}^{\text {out }}\right) \Longrightarrow \operatorname{CallCtx}_{i}\left(\boldsymbol{x}_{i}^{p_{-} \text {in }}, \boldsymbol{x}_{i}^{\text {p_out }}\right)
$$

For instance, a summary for procedure foo in Fig. 11 is $\operatorname{Sum}_{f o o}\left(\left(y, g_{6}\right),\left(r, g_{7}\right)\right)=$ $(y<M A X \Rightarrow r>y) 5$ A (forward) calling context for the first call to procedure foo in main is CallCtx $f_{f o o_{0}}\left(\left(x_{0}, g_{1}\right),\left(x_{1}, g_{2}\right)\right)=\left(g_{1} \Rightarrow x_{0}<0\right)$.

We observe that the guard variables are also used in defining summaries and calling contexts. They have the same meaning as in transition functions. The reason we have defined CallCtx over both input and output parameters is so we can propagate it in forward or backward directions.

\section{Compositional Verification and Refutation Overview}

A decomposition of a verification problem intuitively splits the original problem into a set of sub-problems that cover the original problem. The decomposition operator for the problem has a corresponding composition operator for composing the results obtained from the sub-problems in order to obtain a solution of the original problem.

In terms of program executions, a decomposition can be viewed as a way a proof of verification splits the behaviour, i.e. the set of all execution traces of a program, in constructing the proof. Consider a safe version of the code in Fig. 11 where the assertion in bar is changed to $z \leq 10$. A safety proof for the program can be constructed hierarchically by using the following summaries for foo and bar: $\operatorname{Sum}_{f o o}\left(\left(y, g_{6}\right),\left(r, g_{7}\right)\right)=\left(r=y+1 \wedge g_{6}=g_{7}\right)$ and $\operatorname{Sum}_{\text {bar }}\left(\left(z, g_{8}\right),\left(g_{9}\right)\right)=$ $\left(g_{9} \Rightarrow z \leq 10\right)$. Then, the proof for main can be constructed using the recursive Algorithm 10 The proof for the leaves (foo and bar) involves showing their transition functions imply their respective summary. Proof composition for a non-leaf procedure will use the caller summaries to similarly construct a proof (a summary) for the caller. In our example, the program is indeed proved safe as the algorithm constructs a Sum $_{\text {main }}$, which, in this case, can be a suitable abstraction of the transition function for main, that is not false, while checking that the constructed summaries verify all the embedded properties.

For sequential programs, decompositions can be vertical or horizontal. A vertical decomposition usually focuses on entire execution traces and splits the

${ }^{5}$ MAX denotes the maximum possible value in the type of $y$. 


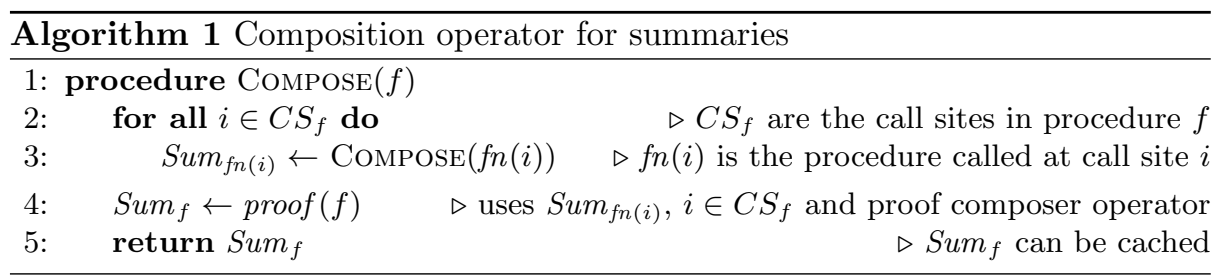

behaviour of the program into subsets of end-to-end traces, e.g. program slicing [16]. A horizontal decomposition is usually based on a syntactic decomposition of the program e.g. into procedures. This paper focuses on solving the refutation problem with horizontal decompositions.

The challenge in automating horizontal compositional verification lies in synthesising a set of precise summary predicates for the procedures in the call graph. Note that in the program in Fig. 1, it was essential to constrain the input $z$ to bar to be $(z \leq 1)$ to get a proof. This effort is made harder if the code has loops, which require invariants and use of abstractions. The calling contexts and summaries can be mutually dependent even for non-recursive programs. In general, one requires iterative fix-point computation on the call-graph structure, possibly using abstraction and refinement. A pre-requisite for performing abstraction refinement is the ability to refute safety and check for spurious counterexamples also in a modular and efficient fashion, which is the goal of this paper.

A Practical View of the Modular Refutation Problem. Consider the example in Figure 1 in Section 2. This program is unsafe because when bar is called the actual argument to it that takes the place of $z$ can at most be only 1 . The question is if we can arrive at this refutation modularly. Analysing procedure bar in isolation indeed gives a counterexample, which could be possibly spurious.

Instantiated on the example in Fig. 1. a refutation involves checking $\neg \forall z, g_{8}$ : $g_{8} \Rightarrow(z>10)$. A counterexample could be $g_{8} \wedge z=5$, for example. The question is now how to decide whether this counterexample is spurious or not, and to find a valid counterexample if one exists. For instance, $z=5$ turns out to be spurious if we consider the whole program because it clashes with $x_{0}<0$ in main. However, $z=-8$ would be a valid counterexample.

The set of local counterexamples found in a procedure $f$ might contain many counterexamples that are spurious for the whole program, i.e. they are infeasible from the entry point of the program. A definite answer to this question cannot be given by looking at the local problems alone, but only by analysing the global one. This is the reason why refutation in horizontal decompositions is hard - unlike refutation in vertical decompositions where a refutation of the local problem implies the refutation of the global one.

Intuitively, the negation of the assertion has to be hoisted up along the error path to the entry point of the program. If the obtained weakest precondition for the violation of the assertion is not false, then the counterexample is feasible. Propagating up the counterexample itself is not sufficient to decide spuriousness as illustrated above. 


\section{Formalising Horizontal Compositional Refutation}

In this section we formalise the problem of safety refutation for sequential programs. To simplify the presentation we focus on loop-free programs. The formalisation for programs with loops is structurally similar, but in addition, requires the handling of invariants, which is orthogonal to the compositional aspect.

We give three different formalisations - the first corresponds to a monolithic approach, and the remaining two correspond to compositional approaches.

\subsection{Monolithic Safety Refutation Problem}

For non-recursive programs, since one can always inline every procedure call at its call site, we can replace every call by recursively inlining its body. Then, to refute safety we have to show unsatisfiability of the following formula:

$$
\forall \hat{X}: \bigwedge_{j \in C S} g_{f_{\text {entry }}^{\text {in }}} \wedge T_{f n(j)}\left(\boldsymbol{x}_{j}^{i n}, \boldsymbol{x}_{j}^{\text {out }}\right) \wedge \text { InlineSums }_{f n(j)} \Rightarrow \operatorname{Props}_{f n(j)}\left(\boldsymbol{x}_{j}\right)
$$

where

- InlineSums ${ }_{f}$ is $\bigwedge_{i \in C S_{f}}$ InlineSum $_{f n(i)}\left(\boldsymbol{x}_{i}^{p_{i} \text { in }}, \boldsymbol{x}_{i}^{p_{-} \text {out }}\right)$,

- InlineSum ${ }_{f}\left(\boldsymbol{x}_{f}^{\text {in }}, \boldsymbol{x}_{f}^{\text {out }}\right)$ is $T_{f}\left(\boldsymbol{x}_{f}^{\text {in }}, \boldsymbol{x}_{f}^{\text {out }}\right) \wedge$ InlineSums $_{f}$,

$-\hat{X}$ is the entirety of variables in (11),

- and the conjunction with $g_{f_{\text {entry }}}^{\text {in }}$ states that the entry procedure is reachable 6 Alternatively, we can write:

$$
\begin{aligned}
\exists & \overbrace{\text { Sum }_{f}, \ldots:}^{\text {for all } f \in F} \bigwedge_{f \in F} \forall X_{f}: \\
& \quad\left(g_{f_{\text {entry }}^{\text {in }}} \wedge T_{f}\left(\boldsymbol{x}_{f}^{\text {in }}, \boldsymbol{x}_{f}^{\text {out }}\right) \wedge \operatorname{Sums}_{f} \Longrightarrow \operatorname{Props}_{f}\left(\boldsymbol{x}_{f}\right)\right) \\
\wedge & \left(T_{f}\left(\boldsymbol{x}_{f}^{\text {in }}, \boldsymbol{x}_{f}^{\text {out }}\right) \wedge S u m s_{f} \Longleftrightarrow \operatorname{Sum}_{f}\left(\boldsymbol{x}_{f}^{\text {in }}, \boldsymbol{x}_{f}^{\text {out }}\right)\right)
\end{aligned}
$$

where $\operatorname{Sums}_{f}$ is $\bigwedge_{i \in C S_{f}} S u m_{f n(i)}\left(\boldsymbol{x}_{i}^{p_{-} \text {in }}, \boldsymbol{x}_{i}^{p_{-} \text {out }}\right)$.

This formulation uses a predicate $S_{u m}$ to exactly express the behaviours of each procedure $f$. (11) and (2) are equisatisfiable, i.e., 1 is satisfiable iff 2 is satisfiable. The existential quantifier in (2) can be uniquely eliminated by recursively replacing the $\operatorname{Sum}_{f}$ predicates by left-hand side of the equivalence in the last line in (2), obtaining (1). Note that solving (1) is NP-complete, whereas solving (2) is PSPACE-complete. However, (11) may be exponentially larger (in the number of variables) than (2).

Both versions are monolithic because they consider the entire program as a whole. In particular, (2) finds summaries globally, i.e. for the whole program.

Also note that, proving unsatisfiability of (2) shows the inexistence of a verification proof, but it does not directly allow us to derive a counterexample in terms of an execution trace because of the universal quantification of the variables. Moreover, showing unsatisfiability of (2) is difficult because it involves proving the inexistence of summary predicates. For this reason, many practical

${ }^{6}$ This amounts to using $T_{f_{\text {entry }}}\left[\right.$ true $\left./ g_{f_{\text {entry }}}^{\text {in }}\right]$ as the transition relation of $f_{\text {entry }}$. 
techniques, such as SAT-based Bounded Model Checking use (1) (considering bounded unwindings for programs with loops in order to make them loop-free). Note that negating (1) results in an existentially quantified problem, whose satisfiability witnesses a refutation in the form of values for the variables $\hat{X}$.

However, solving (1) monolithically is often intractable. Therefore, we want to decompose the problem into smaller subformulae that are faster to solve. (2) is amenable to decomposition, but it does not allow us to approximate the summaries with the help of abstractions (because of $\Leftrightarrow$ in last line). Therefore we give a third formulation of the monolithic problem that additionally uses calling contexts. The calling context for the entry procedure is $g_{f_{\text {entry }}^{i n}}^{\text {. }}$

$$
\begin{aligned}
\exists \overbrace{\text { Sum }_{f}, \text { CallCtx }_{f}, \ldots:}^{\text {for all } f \in F} \bigwedge_{f \in F} & \forall X_{f}: \\
\left(\text { CallCtx } f_{f}\left(\boldsymbol{x}_{f}^{\text {in }}, \boldsymbol{x}_{f}^{\text {out }}\right) \wedge\right. & \operatorname{Props}_{f}\left(\boldsymbol{x}_{f}\right) \wedge \\
T_{f}\left(\boldsymbol{x}_{f}^{\text {in }}, \boldsymbol{x}_{f}^{\text {out }}\right) \wedge \text { Sums }_{f} \Longrightarrow & \operatorname{Sum}_{f}\left(\boldsymbol{x}_{f}^{\text {in }}, \boldsymbol{x}_{f}^{\text {out }}\right) \wedge \\
& \left.\bigwedge_{j \in C S_{f}} \operatorname{CallCtx}_{f n(j)}\left(\boldsymbol{x}_{j}^{p_{\text {in }}}, \boldsymbol{x}_{j}^{p_{-} \text {out }}\right)\right)
\end{aligned}
$$

Eq. (3) is also equisatisfiable with (2), although (3) admits more solutions to $\mathrm{Sum}_{f}$ including those that are over-approximations adequate to prove the properties. To see this, if (2) is satisfiable, the precise solution of (2) for Sum $_{f}$ can be used to satisfy (3) by plugging it in for both CallCtx $f$ and Sum $_{f}$ in (3). If (2) is unsatisfiable, then so is (3) because one or all behaviour included in Sum $_{f}$ solution of (2) violates one of the properties. Then, every solution to (3) would violate the properties as they are over-approximations of the precise summaries.

\subsection{Modular Safety Refutation Problem}

Let us now have a look at the horizontal decomposition following the procedural structure of the program. The goal is to compute the summaries Sum $_{f}$ for each $f$ while considering only $f$ and the summaries for the procedures called in $f$. We can attempt at achieving this by flipping the existential quantifier $\left(\exists S u m_{f}\right)$ and the top-level conjunction $\left(\bigwedge_{f \in F}\right.$ in (3) $)$. However, this does not result in an equisatisfiable formula because existential quantification does not distribute over conjunctions. Therefore, we need an alternative formulation to solve the existential query per procedure. One approach is to search for a minimal solution for summaries and calling contexts occurring within each calling site of procedure $f$ for a given context for $f$ that satisfies all the embedded properties in $f$ as shown in 4 . I.e. for each $f \in F$ we have:

$$
\begin{aligned}
& \min \operatorname{Sum}_{f}, \overbrace{\text { CallCtx }_{j}, \ldots}^{\text {for all } j \in C S_{f}}: \forall X_{f}: \\
& \left(\operatorname{CallCtx}_{f}\left(\boldsymbol{x}_{f}^{\text {in }}, \boldsymbol{x}_{f}^{\text {out }}\right) \wedge\right. \\
& T_{f}\left(\boldsymbol{x}_{f}^{\text {in }}, \boldsymbol{x}_{f}^{\text {out }}\right) \wedge \text { Sums }_{f} \Longrightarrow \text { Props }_{f}\left(\boldsymbol{x}_{f}\right) \wedge \\
& \operatorname{Sum}_{f}\left(\boldsymbol{x}_{f}^{\text {in }}, \boldsymbol{x}_{f}^{\text {out }}\right) \wedge \\
& \left.\bigwedge_{j \in C S_{f}} \operatorname{CallCtx}{ }_{j}\left(\boldsymbol{x}_{j}^{p_{\text {_in }}}, \boldsymbol{x}_{j}^{p_{-} \text {out }}\right)\right)
\end{aligned}
$$


$\min P: F(P)$ is defined w.r.t. implication order for a formula $F$ involving predicates $P$, i.e. as $\exists P: F(P) \wedge \forall P^{\prime}:\left(P^{\prime} \Rightarrow P\right) \Rightarrow \neg F\left(P^{\prime}\right)$. Note that $\min P$ is not unique in a partial order. (4) gives a solution for $\operatorname{Sum}_{f}$ and the calling contexts for all embedded calling sites relative to a CallCtx $f$, assuming there is a minimal solution for all embedded procedures. But, we have not broken the dependency between calling contexts and summaries. Solving this problem requires computing a fixed point in the composition operator (presented below) and computing minimal solutions for the summary and calling context predicates. That is, what has been an existential second-order satisfaction problem in (3), has now become a second-order minimisation $(\exists \forall)$ problem. The reason for this is that the mere existence of a solution for $\operatorname{Sum}_{f}$ and $\operatorname{CallCtx}_{f n(j)}$ does not prove that the overall verification problem holds. Therefore, we pessimistically have to assume that we require the exact calling contexts and summaries in order to decide the problem during proof composition.

The proposed proof composition operator (compose) with calling contexts is shown in Alg. 2 and is more complex than Alg. 11 The idea is to use the call graph of the program to compute the minimal calling context for each call site of procedure call of $f$ piecewise in a top-down fashion use that calling context to compute a piecewise minimal summary for $f$ for that call site (note the conjunction on Line 12 of Alg. 22) consistent with all the properties in $f$. The piecewise summaries and contexts are combined disjunctively as they are built, which takes care of the dependency between summary and calling contexts. In the algorithm, each time compose is called recursively for $f$, it is called with a new piece of entry calling context for $f$ and (4) is solved with summaries computed up to that point for the procedures in the body of $f$. Solving the equation smay result in new contexts for each call site (if any) inside $f$ and a new piece of summary for $f$ all of which are accumulated.

For a program with entry $f_{\text {entry }}$, a proof can be constructed by calling compose $\left(f_{\text {entry }}, g_{f_{\text {entry }}}^{\text {in }}\right)$. The calling context $g_{f_{\text {entry }}}^{\text {in }}$ means that the entry procedure is reachable. The calling context of all embedded functions are initialised to false as that is the least element and also makes everything following the first call site unreachable. The summary for each $f$ is initialised to $\neg g_{f}^{\text {out }}$, meaning that its exit is not reachable and hence execution cannot continue beyond any call to $f$. This initial value for summary has the effect of blocking analysis of all functions following $f$ in the code until a piecewise summary is computed for $f$.

Observe that, as opposed to monolithic (3) where the fixed point computation for resolving the mutually dependent summary and calling context predicates (cf. 23] ) is done within the solver for solving the monolithic formula, the fixed point in the modular version must be computed during the composition of the individual results. I.e. we have to saturate the $\mathrm{Sum}_{f}$ and $\mathrm{CallCtx}_{f}$ predicates.

Theorem 1. We obtain Sum $f_{\text {entry }}=$ false using Alg. 20 iff (3) is unsatisfiable. I.e. horizontal decomposition is sound and complete.

Proof (sketch): We prove this by induction on the depth $(k)$ of the top-level function in the call graph of the program. 


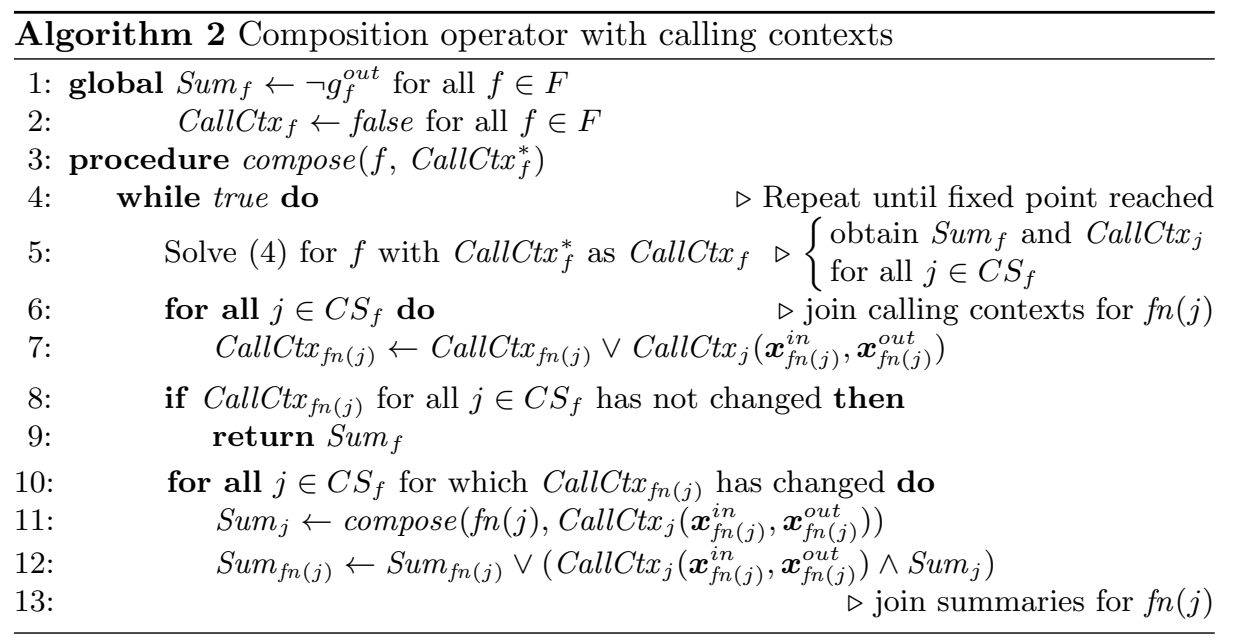

For the base case $(k=0)$, there is only one procedure call - the call to the entry procedure, $f_{\text {entry }}$. Since the calling context of $f_{\text {entry }}$ is $g_{f_{\text {entry }}}^{\text {in }}$, and there are no other procedure calls, it is evident that computing Sum $_{f_{\text {entry }}}$ from Alg. 2 effectively reduces to finding it by solving (4) (line 5 of Alg. 2), with Sums and CallCtx $_{j}$ not present. This makes Eq. (41) and (3) identical and hence the theorem follows trivially.

Proceeding with the induction step for $k+1$ assuming the theorem holds for all functions in the call graph with depth $\leq k$. That is, we assume as hypothesis the summary computed by compose satisfies theorem for all function calls in the body of $f$ for all contexts. Suppose (3) is unsatisfiable. We will argue that Alg. 2 must return false.

If (3) is unsatisfiable then there must be at least one function (either the top-level function or something deeper in the call graph) that is unsatisfiable. Suppose it is one of the called functions, say $h$, that is unsatisfiable. Then, by our induction hypothesis, the algorithm will return false for Sum $_{h}$. The moment one of the embedded summaries becomes false our algorithm immediately saturates because Alg. 2 is trivially satisfiable with minimal solution of false for Sum $_{f}$. If (3) is unsatisfiable because the top-level function $f$ is unsatisfiable, then it must be because Props $_{f}$ is inconsistent with $T_{f}$. In this case, Alg. 2 can only return false.

\subsection{Modular Safety Refutation with Witnesses}

(44) suffers from the same problem as (3) that we cannot extract counterexamples in terms of an execution trace in case of a refutation because the formulae are unsatisfiable for refutations. Therefore we give next a formulation and a corresponding composition operator that produces refutation witnesses. The idea here is to compute piecewise contexts and summaries backwards starting from exit points of each procedure, much like a weakest-precondition computation 
works. Additionally, we start with negation of properties and compute maximal summary and contexts that possibly lead the program to an error state. In other words, a summary computed for $f$ represent maximal symbolic witness to all the states reachable to safety violation. Such a summary can be obtained as maximal solutions to the equation shown in 5

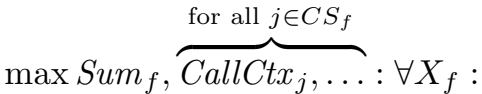

$$
\begin{aligned}
& \operatorname{Sum}_{f}\left(\boldsymbol{x}_{f}^{\text {in }}, \boldsymbol{x}_{f}^{\text {out }}\right) \wedge \\
& \bigwedge_{j \in C S_{f}} \operatorname{CallCtx}_{j}\left(\boldsymbol{x}_{j}^{\text {p-in }}, \boldsymbol{x}_{j}^{\text {pout }}\right) \Longrightarrow\left(\text { CallCtx }_{f}\left(\boldsymbol{x}_{f}^{\text {in }}, \boldsymbol{x}_{f}^{\text {out }}\right) \vee \neg \operatorname{Props}_{f}\right) \wedge \\
& T_{f}\left(\boldsymbol{x}_{f}^{\text {in }}, \boldsymbol{x}_{f}^{\text {out }}\right) \wedge \text { Sums }_{f}
\end{aligned}
$$

where $\max P . F(P)$ is defined as usual: $\exists P . F(P) \wedge \forall P^{\prime} .\left(P \Rightarrow P^{\prime}\right) \Rightarrow \neg F\left(P^{\prime}\right)$.

(51) describes maximal solutions for the summary and calling contexts that are contained in the behaviour of the procedure. That is the reason the predicates for the summary and the calling contexts (for the called functions) appear on the left-hand side of the implication and the transition relation is on the right-hand side, i.e. reversed in comparison with (44). The disjuncts in the first part of the consequent of (5) are the sources of safety violations: these are safety violations in the caller (which are propagated by CallCtx $_{f}$ ), and safety violations in $f$ itself $\left(\neg\right.$ Props $\left._{f}\right)$. Safety violations in callees are propagated through the summaries. Both these are constrained to be consistent with the transition relation of $f$ (with current summaries plugged in for the called functions), which ensures spurious errors are not propagated upwards.

We use the composition operator as in Alg. 2. but with the following modifications to the initialization. We call this composition operator compose or Alg. 2] from now on.

- Initially, Sum $_{f} \leftarrow \neg g_{f}^{\text {in }}$ for all $f \in F$, meaning that the entry of $f$ is not backwards-reachable.

- In Line 5, we solve (5).

The calling contexts for all embedded functions are initialized to false as before except for the top-level function $f_{\text {entry }}$. A refutation is constructed by computing compose $\left(f_{\text {entry }}, \neg g_{f_{\text {entry }}^{\text {out }}}\right)$. The calling context $\neg g_{f_{\text {entry }} \text { out }} f_{\text {entry }}$ means that we cannot reach the regular exit of the entry procedure if there is a property violation. If there are no property violations at this level (or no properties), then this choice for top-level context would still work as the second conjunct in equation 5. which denotes the transition relation, would ensure the precise contexts propagated to the first embedded call site from exit point. The choice of initial summary of $\neg g_{f}^{i n}$ for all embedded functions will ensure that the summaries are generated in order of dependency of function calls backward from the exit point.

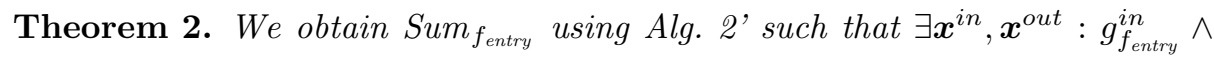
Sum $_{f_{\text {entry }}}\left(\boldsymbol{x}^{\text {in }}, \boldsymbol{x}^{\text {out }}\right)$ iff (3) is unsatisfiable.

Note that the conjunction with $g_{f_{\text {entry }}}^{\text {in }}$ projects the summary on the inputs, which must be satisfiable to have a refutation. 
Proof (sketch): In contrast to Alg. 22 with (4), Alg. 20 uses (5) that computes the maximal solutions for the summary and calling contexts contained in the program behaviour. The summaries and calling contexts are computed such that their projection on the input variables of a procedure is the weakest precondition w.r.t. the properties (Props), whose complement is the refutation. Thus at the entry function, $f_{\text {entry }}$, we get $\operatorname{Sum}_{f_{\text {entry }}}\left(\boldsymbol{x}^{\text {in }}, \boldsymbol{x}^{\text {out }}\right)$ as weakest precondition for the negation of the property such that $g_{f_{\text {entry }}}^{\text {in }} \wedge \operatorname{Sum}_{f_{\text {entry }}}\left(\boldsymbol{x}^{\text {in }}, \boldsymbol{x}^{\text {out }}\right)$ is satisfiable iff (3) is unsatisfiable.

\subsection{Worked Example}

Let us consider the example in Fig. [1, but with the conditional in line 2 being $x<$ 10. We start with $\operatorname{Sum}_{\text {main }}\left(\left(x_{0}, g_{0}\right),\left(g_{5}\right)\right)=\neg g_{0}, \operatorname{Sum}_{\text {foo }}\left(\left(y, g_{6}\right),\left(r, g_{7}\right)\right)=\neg g_{6}$, $\operatorname{Sum}_{\text {bar }}\left(\left(z, g_{8}\right),\left(g_{9}\right)\right)=\neg g_{8}$, and CallCtx $x_{\text {main }}\left(\left(x_{0}, g_{0}\right),\left(g_{5}\right)\right)=\neg g_{5}$, CallCtx $_{\text {foo }}((y$, $\left.\left.g_{6}\right),\left(r, g_{7}\right)\right)=$ false, CallCtx $x_{\text {bar }}\left(\left(z, g_{8}\right),\left(g_{9}\right)\right)=$ false.

The composition operator is called for main. We solve (5):

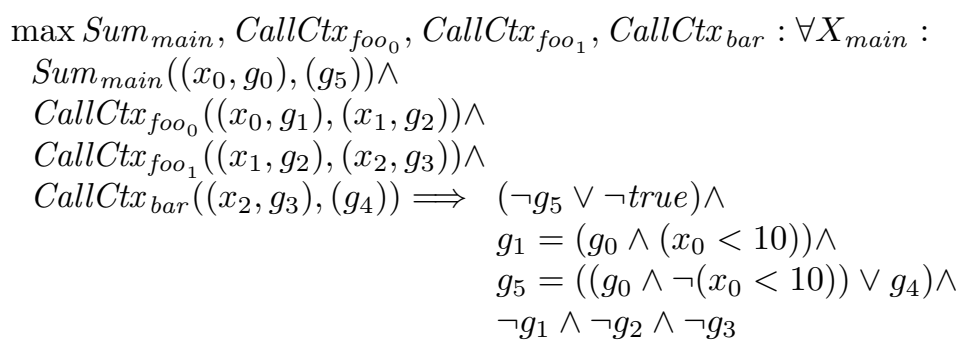

We obtain the following solutions for the predicates: CallCtx $x_{b a r}=\neg g_{4}$, CallCtx $f_{f o o_{1}}=$ $\neg g_{3}$, CallCtx $_{\text {foo }_{0}}=\neg g_{2}$, Sum main $=\neg g_{0} \wedge \neg g_{5}$.

Then we recur into bar with (5) instantiated as:

$$
\begin{aligned}
\max _{S_{\text {bum }}}: \forall z, g_{8}, g_{9}: & \\
\operatorname{Sum}_{\text {bar }}\left(\left(z, g_{8}\right),\left(g_{9}\right)\right) \Longrightarrow & \left(\neg g_{9} \vee \neg\left(g_{8} \Rightarrow z>10\right)\right) \wedge \\
& \left(g_{9}=\left(g_{8} \wedge z>10\right)\right)
\end{aligned}
$$

Hence, we get for $\operatorname{Sum}_{\text {bar }}:\left(g_{8} \Rightarrow \neg(z>10)\right) \wedge \neg g_{9}$.

In Line 6 of Alg. 2, (5) for main is then:

$$
\begin{aligned}
& \max \text { Sum }_{\text {main }}, \text { CallCtx }_{f_{o_{0}}}, \text { CallCtx }_{f_{f o o_{1}}}, \text { CallCtx }_{\text {bar }}: \forall X_{\text {main }} \text { : } \\
& \operatorname{Sum}_{\text {main }}\left(\left(x_{0}, g_{0}\right),\left(g_{5}\right)\right) \wedge \\
& \text { CallCtx }_{f_{o o_{0}}}\left(\left(x_{0}, g_{1}\right),\left(x_{1}, g_{2}\right)\right) \wedge \\
& \text { CallCtx }_{f o o_{1}}\left(\left(x_{1}, g_{2}\right),\left(x_{2}, g_{3}\right)\right) \wedge \\
& \text { CallCtx }_{\text {bar }}\left(\left(x_{2}, g_{3}\right),\left(g_{4}\right)\right) \Longrightarrow\left(\neg g_{5} \vee \neg \text { true }\right) \wedge \\
& g_{1}=\left(g_{0} \wedge\left(x_{0}<10\right)\right) \wedge \\
& g_{5}=\left(\left(g_{0} \wedge \neg\left(x_{0}<10\right)\right) \vee g_{4}\right) \wedge \\
& \neg g_{1} \wedge \neg g_{2} \wedge \\
& \left(g_{3} \Rightarrow \neg\left(x_{2}>10\right)\right) \wedge \neg g_{4}
\end{aligned}
$$


which results in CallCtx $x_{\text {bar }}=\neg g_{4}$, CallCtx $_{f_{o o_{1}}}=g_{3} \Rightarrow \neg\left(x_{2}>10\right)$, CallCtx $f_{f_{o o}}=$ $\neg g_{2}$, Sum $_{\text {main }}=\neg g_{5}$. Hence, CallCtx $x_{\text {foo }}$ is updated to $g_{7} \Rightarrow \neg(r>10)$.

In the next iteration of compose (main) we recur into $f o o_{1}$ and solve:

$$
\begin{aligned}
& \max _{\operatorname{mam}_{f o o}: \forall y, g_{6}, r, g_{7}:} \\
& \operatorname{Sum}_{f o o}\left(\left(y, g_{6}\right),\left(r, g_{7}\right)\right) \Longrightarrow\left(\left(g_{7} \Rightarrow \neg(r>10)\right) \vee \neg \text { true }\right) \wedge \\
&\left(g_{6}=g_{7}\right) \wedge(r=y+1)
\end{aligned}
$$

Thus, $\operatorname{Sum}_{f o o}$ is updated to $\left(g_{6} \Rightarrow \neg(r>10) \wedge g_{7}\right) \wedge(r=y+1)$.

Then in Line 6 in compose(main), we solve

$$
\begin{aligned}
& \max \operatorname{Sum}_{\text {main }}, \text { CallCtx }_{f_{f o o_{0}}}, \text { CallCtx }_{f_{\text {oo }}}, \text { CallCtx }_{\text {bar }}: \forall X_{\text {main }} \text { : } \\
& \operatorname{Sum}_{\text {main }}\left(\left(x_{0}, g_{0}\right),\left(g_{5}\right)\right) \wedge \\
& \text { CallCtx }_{f_{o o_{0}}}\left(\left(x_{1}, g_{2}\right)\right) \wedge \\
& \text { CallCtx } f_{f o o_{1}}\left(\left(x_{2}, g_{3}\right)\right) \wedge \\
& \text { CallCtx }_{\text {bar }}\left(\left(g_{4}\right)\right) \Longrightarrow \quad\left(\neg g_{5} \vee \neg \text { true }\right) \wedge \\
& g_{1}=\left(g_{0} \wedge\left(x_{0}<10\right)\right) \wedge \\
& g_{5}=\left(\left(g_{0} \wedge \neg\left(x_{0}<10\right)\right) \vee g_{4}\right) \wedge \\
& \left(g_{1} \Rightarrow \neg\left(x_{1}>10\right) \wedge g_{2}\right) \wedge\left(x_{1}=x_{0}+1\right) \wedge \\
& \left(g_{2} \Rightarrow \neg\left(x_{2}>10\right) \wedge g_{3}\right) \wedge\left(x_{2}=x_{1}+1\right) \wedge \\
& \left(g_{3} \Rightarrow \neg\left(x_{2}>10\right)\right) \wedge \neg g_{4}
\end{aligned}
$$

which gives us Sum $_{\text {main }}=\left(g_{0} \Rightarrow \neg\left(x_{0}>8\right)\right) \wedge \neg g_{5}$. The calling contexts CallCtx $_{\text {bar }}=\neg g_{4}$, CallCtx $_{f_{o o}}=g_{2} \Rightarrow \neg\left(x_{1}>10\right)$, and CallCtx foo $_{1}=g_{3} \Rightarrow$ $\neg\left(x_{2}>10\right)$ do not result in an update of the calling contexts for foo and bar (Line 8 in Alg. 2). $g_{0} \wedge$ Sum $_{\text {main }}$ is satisfiable, hence, $x \leq 8$ is a (maximal) refutation witness.

\section{Examples of Refutation Algorithms}

Alg. 2 is not only applicable to straight-line programs with multiple procedure invocations, it can still be used for programs with loops by introducing invariants into the formula for the modular subproblem (5). However, in general it is hard to solve the problems without using approximations by bounding the number of unwindings and/or using abstractions for computing the predicates involved.

In the previous section, we have described the elements necessary for compositional, horizontal refutation proofs. In this section, we will give three examples of algorithms that instantiate this framework (Alg. 2), which we have implemented to compare them experimentally in Section 6. We assume that loops have been unwound a finite number of times before application of these techniques. The difference in the following three techniques lies in the abstractions that are used to solve for $\operatorname{Sum}_{f}$ and CallCtx $_{f}$ in (5). We consider techniques that use constraint solving to find counterexamples.

\subsection{Concrete Backward Interpretation}

This technique is the one sketched in the example at the beginning of Section 3 . Formally, we use the domain of predicates that track a single constant value for 
each variable, defined as follows: Let $P(\boldsymbol{x})=\{$ false $\} \cup\left\{\boldsymbol{x}=\boldsymbol{d} \mid d_{i} \in \operatorname{Dom}\left(x_{i}\right)\right\}$ with the domain $\operatorname{Dom}\left(x_{i}\right)$ of variable $x_{i}$, then we admit the following predicates for summaries and calling contexts: $\operatorname{Sum}_{f} \in\left\{g_{f}^{i n} \Rightarrow p \mid p \in P\left(\boldsymbol{x}_{f}^{i n}\right)\right\}$ and CallCtx $_{f} \in\left\{g_{f}^{\text {out }} \Rightarrow p \mid p \in P\left(\boldsymbol{x}_{f}^{\text {out }}\right)\right\}$. We explain now in an example how Alg. 22 proceeds using this domain.

Example. Let us consider the example in Figure 1 in Section 2 We start with compose' $\left(\right.$ main, $\left.\neg g_{5}\right)$. We obtain the calling contexts $\neg g_{2}, \neg g_{3}, \neg g_{4}$ for $f o o_{0}, f o o_{1}$, bar, respectively. We recur into compose' $\left(\right.$ bar,$\left.\neg g_{9}\right)$. We have to solve (5) where $\sum_{b a r}$ is instantiated with the above domain:

$$
\begin{aligned}
\exists d: \forall z, g_{8}, g_{9}: & \\
\left(g_{8} \Rightarrow z=d\right) \Longrightarrow & \left(\neg g_{9} \vee \neg\left(g_{8} \Rightarrow(z>10)\right)\right) \wedge \\
& \left(g_{9}=\left(g_{8} \wedge z>10\right)\right)
\end{aligned}
$$

The partial order of our domain has only two levels false and the values for $\boldsymbol{d}$. Hence, we can implement max by $\exists \boldsymbol{d}$; if there is no $\boldsymbol{d}$ then $p=$ false. A constraint solver may return, for example, $d=-4 ;$ Sum $_{\text {bar }}$ is hence $g_{8} \Rightarrow(z=-4)$. This is an under-approximative summary of bar w.r.t. property violation.

In the next iteration of compose' (main, $\left.\neg g_{5}\right)$ we solve:

$$
\begin{aligned}
& \exists d_{0}, \ldots, d_{3}: \forall x_{0}, g_{0}, \ldots, g_{5}: \\
&\left(g_{0} \Rightarrow x_{0}=d_{0}\right) \wedge \\
&\left(g_{2} \Rightarrow x_{1}=d_{1}\right) \wedge \\
&\left(g_{3} \Rightarrow x_{2}=d_{2}\right) \wedge \\
&\left(g_{4} \Rightarrow d_{3}\right) \Longrightarrow\left(\neg g_{5} \vee \neg \text { true }\right) \wedge \\
& g_{1}=\left(g_{0} \wedge\left(x_{0}<10\right)\right) \wedge \\
& g_{5}=\left(\left(g_{0} \wedge \neg\left(x_{0}<10\right)\right) \vee g_{4}\right) \wedge \\
& \neg g_{1} \wedge \neg g_{2} \wedge \\
&\left(g_{3} \Rightarrow\left(x_{2}=-4\right)\right) \wedge \neg g_{5}
\end{aligned}
$$

and obtain CallCtx $_{f_{0 o_{1}}}=\left(g_{3} \Rightarrow\left(x_{2}=-4\right)\right)$. compose $^{\prime}\left(\right.$ foo $\left._{1} g_{7} \Rightarrow(r=-4)\right)$ returns $g_{6} \Rightarrow(y=-5)$ for Sum $_{f o o_{1}}$. Note that the boolean variable $d_{3}$ stands for the reachability of the exit of bar. Since bar has no return value, this is how its exit is encoded. Proceeding similarly we get compose $\left(\right.$ foo, $\left.g_{7} \Rightarrow(r=-5)\right)=\left(g_{6} \Rightarrow\right.$ $(y=-6))$; and finally $\operatorname{Sum}_{\operatorname{main}}=\left(g_{0} \Rightarrow x_{0}=-6\right)$. Hence, we have found a true global counterexample.

\subsection{Abstract Backward Interpretation}

Abstract backward interpretation computes sufficient preconditions to safety violations, i.e. negations of necessary preconditions to safety. The size of the summaries can vary from very concise to larger than the procedure, depending on the abstraction.

There are a couple of techniques to implement such abstract interpretations that are distinguished by the way abstract preconditions are inferred, e.g. (classical) abstract domain transformers (e.g. [20]), template-based synthesis (e.g. [15]) or interpolation (e.g. [1]). 
We are going to use the template-based synthesis technique used in [3] to solve (5). We know how to compute over-approximative abstractions with that technique. Hence, we use an over-approximation to compute an under-approximation (similar to computing max $f$ by $-\min (-f)$ ). This means we compute predicates $\operatorname{Sum}_{f}^{\tilde{u}}$ and CallCtx ${ }_{j}^{\tilde{u}}$ whose negations are Sum $_{f}$ and CallCtx $_{j}$, respectively. This is done by solving the following formula in place of (5) in Alg. 2].

$$
\begin{aligned}
& \min \operatorname{Sum}_{f}^{\tilde{u}}, \overbrace{\text { CallCtx } x_{j}^{\tilde{u}}, \ldots}^{\text {for all }}: \forall X_{f}: \\
& \left(\operatorname{CallCtx}_{f}^{\tilde{u}}\left(\boldsymbol{x}_{f}^{i n}, \boldsymbol{x}_{f}^{\text {out }}\right) \wedge \operatorname{Sums}_{f}^{\tilde{u}} \wedge\right. \\
& T_{f}\left(\boldsymbol{x}_{f}^{\text {in }}, \boldsymbol{x}_{f}^{\text {out }}\right) \wedge \operatorname{Props}_{f}\left(\boldsymbol{x}_{f}\right) \Longrightarrow \operatorname{Sum}_{f}^{\tilde{u}}\left(\boldsymbol{x}_{f}^{\text {in }}, \boldsymbol{x}_{f}^{\text {out }}\right) \wedge \\
& \left.\bigwedge_{j \in C S_{f}} \operatorname{CallCtx}{ }_{j}^{\tilde{u}}\left(\boldsymbol{x}_{j}^{p_{-} \text {in }}, \boldsymbol{x}_{j}^{p_{\text {_out }}}\right)\right)
\end{aligned}
$$

This formula is derived from (5) by negating ( CallCtx $f \vee \neg$ Props) on the righthand side of (5), which yields (CallCtx $x_{f}^{\tilde{u}} \wedge$ Props), reversing the implication, and minimising to obtain an over-approximation for Sum ${ }^{\tilde{u}}$ and CallCtx ${ }^{\tilde{u}}$. Similar approaches are used in [10/5]. Since convex domains are too imprecise for this purpose, we use a disjunctive domain $[22$. For our experiments we used intervals as a base domain. Formally, let $P(\boldsymbol{x})=\left\{\bigvee_{k} \boldsymbol{d}_{k}^{\prime} \leq \boldsymbol{x} \leq \boldsymbol{d}_{k} \mid d_{i}, d_{i}^{\prime} \in \operatorname{Dom}\left(x_{i}\right), k \geq 0\right\}$, then Sum $_{f} \in\left\{g_{f}^{\text {in }} \Rightarrow p \mid p \in P\left(\boldsymbol{x}_{f}^{\text {in }}\right)\right\}$ and CallCtx $f \in\left\{g_{f}^{\text {out }} \Rightarrow p \mid p \in P\left(\boldsymbol{x}_{f}^{\text {out }}\right)\right\}$. Our implementation also ensures that arithmetic overflows create new disjuncts in order to avoid precision loss. The second source of additional disjuncts that we take into account are Lines 7 and 12 in Alg. 2].

Example. For the example in Figure 1 we compute compose $\left(\right.$ main, $\left.\neg g_{5}\right)$. We solve (8) with CallCtx main $^{\tilde{u}}=g_{5}$ and get CallCtx ${ }_{\text {bar }}^{\tilde{u}}=g_{4}$, i.e. CallCtx bar $_{\text {ball }}=\neg g_{4}$.

We recur into compose $\left(\right.$ bar, $\left.\neg g_{9}\right)$, i.e. CallCtx $x_{b a r}^{u}=g_{9}$ We have to solve (8) instantiated with our domain.

$$
\begin{aligned}
& \exists d, d^{\prime}: \forall z, g_{8}, g_{9}: \\
& \quad\left(g_{9} \wedge \text { true } \wedge\right. \\
& \left.\quad\left(g_{9}=\left(g_{8} \wedge z>10\right)\right) \wedge\left(g_{8} \Rightarrow(z>10)\right) \Longrightarrow\left(g_{8} \Rightarrow\left(d \leq z \leq d^{\prime}\right)\right)\right)
\end{aligned}
$$

Note that $\operatorname{Sums} s_{f}^{\tilde{u}}$ is true because the initial under-approximations are false - the superscript $\tilde{u}$ flags predicates that carry negations of under-approximations. We get $\operatorname{Sum}_{\text {bar }}^{\tilde{u}}=\left(g_{8} \Rightarrow(11 \leq z \leq M A X)\right)$, i.e. $\operatorname{Sum}_{\text {bar }}=\left(g_{8} \wedge(M I N \leq z \leq 10)\right)$. $M A X$ and $M I N$ denote the maximum, resp. minimum, possible value for the type of $z$.

We proceed similarly. Finally, compose $\left(\operatorname{main}, \neg g_{5}\right)$ computes $S_{u m}^{\tilde{u}}{ }_{\text {main }}=$ $\left(g_{0} \Rightarrow\left(9 \leq x_{0} \leq M A X\right)\right)$, i.e. Sum $_{\text {main }}=\left(g_{0} \wedge\left(M I N \leq x_{0} \leq 8\right)\right)$.

Note that (8) expresses an over-approximation of good states; the complement is therefore guaranteed not to contain any good states, but only bad and unreachable states, and hence no strict under-approximation of bad states. However, this does not matter since we project $S u m_{f_{\text {entry }}}$ on the initial condition (see Thm. 2) to obtain a true under-approximation of inputs that violate a property. 
Abstract backward interpretation is not limited to bounded unwindings of the transition relation, but can also be used for programs with loops (cf. [115]) by calling invariants into play in (8) .

\subsection{Symbolic Backward Interpretation}

This technique computes the exact weakest precondition for the bounded problem. The technique is complete for loop-free programs. However, the size of the obtained summaries is in the same order as the procedure size in the worst case.

The domain used are sets of variables, so-called dependency sets. These sets of variables, $X_{f}^{\text {in }}, X_{f}^{\text {out }}, X_{j}^{p_{\text {_in }}}, X_{j}^{p_{-} \text {out }}$, describe which variables should be kept as relevant part of the summary. We then use them to compute an exact summary as the following predicate $\operatorname{Sum}_{f}\left(\boldsymbol{x}^{\text {in }}, \boldsymbol{x}^{\text {out }}\right)$ :

$$
\begin{aligned}
& \exists X_{f} \backslash(X_{f}^{\text {in }} \cup X_{f}^{\text {out }} \cup \overbrace{\left.X_{j}^{p_{-} \text {in }} \cup X_{j}^{p_{-} \text {out }} \cup \ldots\right)}^{\text {for all } j \in C S_{f}}: \\
& \quad\left(\text { CallCtx }_{f}\left(\boldsymbol{x}^{\text {in }}, \boldsymbol{x}^{\text {out }}\right) \vee \neg \text { Props }_{f}\right) \wedge T_{f}\left(\boldsymbol{x}^{\text {in }}, \boldsymbol{x}^{\text {out }}\right) \wedge S u m s_{f}
\end{aligned}
$$

We implement the existential quantification in (10) by Gaussian elimination to eliminate as many of the intermediate or irrelevant variables as possible. After elimination the summary contains only variables that have a dependency on the property Props $_{f}$, on $\boldsymbol{x}^{\text {out }}$, or on the placeholder predicates, which are going to be replaced by summaries during the composition. The elimination can have positive and negative effects on the formula size depending on non-determinism and control flow paths in the procedure.

The composition operator is the same horizontal composition operator as in the two previous techniques. Context-sensitivity is exploited exactly in the same way as in the previous two techniques. The calling context at call site $j$ is the set of output variables $X_{j}^{p_{\text {_out }}}$ that a procedure call backward-transitively depends on the given property. The resulting calling context dependency set $X_{f}^{\text {out }}$ is then used for eliminating intermediate variables in (10) in addition to the dependency sets obtained from Sums $_{f}$, and Props $_{f}$. The set of input variables $X_{f}^{\text {in }}$ that have not been eliminated is the dependency set $X_{f}^{p_{-} i n}$ of the summary $\operatorname{Sum}_{f}$.

Any satisfiable assignment to $\boldsymbol{x}_{f_{\text {entry }}}^{\text {in }}$ in the formula obtained by Gaussian elimination of the summary predicates in the entry function is a feasible global refutation.

Example. For example, in Fig. 11 the symbolic backward interpreter starts from the exit of main with $X_{\text {main }}^{\text {out }}=\emptyset$ to start with. As it arrives in bar, it retains the negation of the assertion $\neg\left(g_{8} \Rightarrow(z>10)\right)$ and updates the dependency set to $X_{b a r}^{i n}=\left\{z, g_{8}\right\}$. On simplification, this gives the summary for bar as $g_{8} \wedge \neg(z>10)$.

Then the technique proceeds to the caller of bar, replacing the variables in the dependency set by the parameter passed, i.e. $X_{\text {foo }_{1}}^{p_{-} \text {ut }}=\left\{x_{2}, g_{3}\right\}$. Then it recurs into the call to foo. The statement $r=y+1$ gives the summary of foo as 
$r=y+1$ and the dependency set $\left\{y, g_{6}\right\}$. The next call to foo has already been analysed with the same dependency set, hence there is no need to recur.

Proceeding in the main function, we finally get the summary for main as $\left(g_{1}=\right.$ $\left.g_{0} \wedge\left(x_{0}<0\right)\right) \wedge$ foo $_{0}\left(\left(x_{0}, g_{1}\right),\left(x_{1}, g_{2}\right)\right) \wedge$ foo $_{1}\left(\left(x_{1}, g_{2}\right),\left(x_{2}, g_{3}\right)\right) \wedge \operatorname{bar}\left(\left(x_{2}, g_{3}\right),\left(g_{4}\right)\right)$. Substituting the placeholder predicates by their respective summaries (variables are renamed) allows us to evaluate the summary for main. Since it is satisfiable, we have found a global refutation.

\section{Experiments}

We performed a number of experiments to evaluate compositional refutation techniques in comparison with monolithic approaches.

Implementation We have implemented these safety refutation techniques as an extension to 2LS 243]. $2 \mathrm{LS}$ is a verification tool built on the CPROVER framework 9, using MiniSAT 2.2.1 as the backend solver (although other SAT and SMT solvers with incremental solving support can also

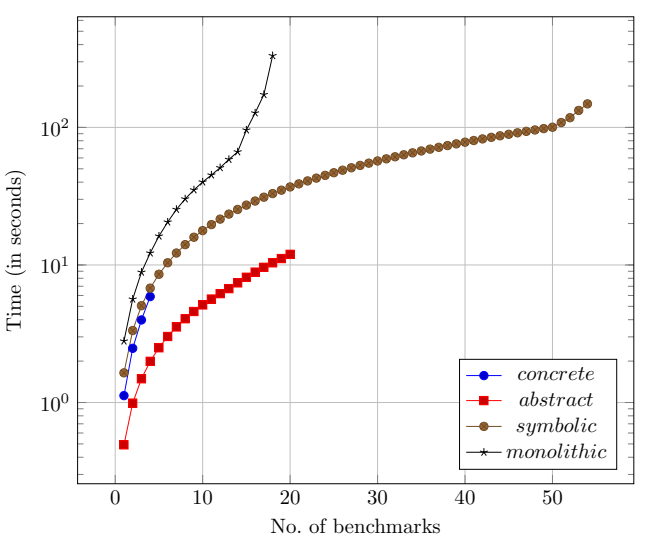

Fig. 2. Comparison on product-lines benchmarks be used). We limit resources to 900 seconds CPU time and 13 GB memory per benchmark. To aid reproducibility, we provid 7 the implementation sources along with the compilation instructions, the benchmarks, and scripts that can be used to run the tool on the benchmarks. As explained in Section 5] the three techniques are instances of a context-sensitive inter-procedural analysis that traverses the callgraph backwards and propagates summaries and calling contexts. For the concrete interpretation, values for non-deterministic choices are picked by the SAT solver. For the abstract interpretation we use disjunctions of intervals.

Benchmarks We selected the unsafe examples (265 benchmarks) from the productlines collection of the SV-COMP 2017 benchmarks set for our experiments. These benchmarks have a reasonably complex procedural structure (83 procedures per benchmark on average), which makes it suitable to test the effectiveness of our techniques. We set an unwinding depth of 5 for all the benchmarks, across all the techniques. The chosen depth might have been, in some cases, higher than what would be necessary to find a refutation. However, the aim of our experiments was to compare the scalability of the techniques in general, and not to find out the least amount of time needed to solve a given benchmark.

7 https://github.com/kumarmadhukar/2ls/tree/atva17 
Results Fig. 2 shows the results plotting for each technique the cumulative time (y-axis) it takes to solve the given number of benchmarks (x-axis). The longer the line for a technique extends to the right the more benchmarks were solved within the resource limits. These results show some interesting tendencies. We observe that the symbolic backward interpretation performs best. It is complete, but could potentially degrade into a monolithic analysis if summaries cannot be sufficiently simplified and reused. But on this benchmark set it works quite well on a certain number of benchmarks. The abstract backward interpretation is very fast on a couple of benchmarks, but then remains inconclusive. This is supposedly due to the imprecision introduced by the weak abstract domain that we use. Yet, this is encouraging that by a clever choice of abstractions one could outperform the symbolic backward interpretation. The concrete backward interpretation succeeds only on very few benchmarks and is surprisingly slow. An explanation for this is that it is required to make non-deterministic choices that may turn out to be bad choices and make a counterexample infeasible. Moreover, the summaries that it computes usually do not generalise beyond the procedure invocation they were generated for. Hence, this technique is likely to degrade into following the entire execution path, spoiling the benefits of modularity while exhibiting the drawbacks of abstraction. The monolithic analysis (BMC), which is based on full inlining is slowest but solves almost as many benchmarks as the abstract one.

\section{Conclusion}

We investigated compositional refutation techniques in horizontal, e.g. proceduremodular, decompositions of sequential programs. We showed how to derive a compositional refutation framework step by step from the monolithic problem. We also compared the completeness properties of concrete, abstract and symbolic modular refutation approaches. Our experiments show that compositional refutation techniques have an advantage over monolithic approaches, however, not all tested approaches perform equally well because of their varying completeness. Using a portfolio of fast incomplete techniques and slower complete ones may ensure that modular techniques are always at least as fast as monolithic ones in practice.

Open questions Modular analyses should be independent of a program's syntactic structure because real-world programs are not written in a nice and balanced way that would enable efficient modular analysis. It would be worthwhile to explore semantic decompositions into modules in order to make these techniques scale on real-world programs. W.r.t. the inter-procedural backward analysis, it remains to be investigated how to handle recursion.

Moreover, it would be interesting to look into compositional refutation in termination analysis. Also there, spuriousness of local refutations can occur due to lack of context information: To find a counterexample to termination one needs to find a stem from the entry point. Compositionality in this context has 
been explored in the Ultimate tool 17. We would also consider performance comparisons with testing, i.e. dynamic refutation techniques (random, directed, concolic, etc.) to be beneficial to advance research in static refutation techniques.

Related work Compositional automated verification approaches have been considered in the tools Whale 11 and FunFrog [26, for example. Horn clause encodings were used in [18. These tools eventually use interpolation to compute abstractions. Under-approximating precondition inference techniques have been proposed for polyhedra 20] and with the help of bit blasting and loop iteration estimation [4]. All these techniques can be used in our setting, however, their completeness properties remain to be evaluated. Completeness considerations [21] have been conducted for compositional LTL model checking [8]7 of (parallel) compositions of (infinite-) state transition systems. Since the decomposition of sequential programs can be encoded into a composition of parallel programs (with appropriate synchronisation), their completeness results are expected to hold in our setting. Compositionality has also been explored in the context of dynamic test generation to achieve scalability by memoizing symbolic execution sub-paths as test summaries [13. This has given rise to an incremental approach for statically validating symbolic test summaries against code changes [14. In our framework memoization is naturally handled by the composition operator.

\section{References}

1. Albarghouthi, A., Gurfinkel, A., Chechik, M.: Whale: An interpolation-based algorithm for interprocedural verification. In: Verification, Model Checking, and Abstract Interpretation. LNCS, vol. 7148, pp. 39-55. Springer (2012)

2. Alur, R., de Alfaro, L., Henzinger, T.A., Mang, F.Y.C.: Automating modular verification. In: Concurrency Theory. pp. 82-97 (1999)

3. Brain, M., Joshi, S., Kroening, D., Schrammel, P.: Safety Verification and Refutation by $k$-Invariants and $k$-Induction. In: Static Analysis Symposium. LNCS, vol. 9291, pp. 145-161. Springer (2015)

4. Brauer, J., Simon, A.: Inferring definite counterexamples through underapproximation. In: NASA Formal Methods. LNCS, vol. 7226, pp. 54-69 (2012)

5. Chen, H., David, C., Kroening, D., Schrammel, P., Wachter, B.: Synthesising interprocedural bit-precise termination proofs. In: Automated Software Engineering. pp. 53-64. ACM (2015)

6. Clarke, E.M., Grumberg, O., Jha, S., Lu, Y., Veith, H.: Counterexample-guided abstraction refinement. In: Computer-Aided Verification. LNCS, vol. 1855, pp. 154169. Springer (2000)

7. Clarke, E.M., Grumberg, O., Long, D.E.: Model checking and abstraction. Transactions on Programming Languages and Systems 16(5), 1512-1542 (1994)

8. Clarke, E.M., Long, D.E., McMillan, K.L.: Compositional model checking. In: Logic in Computer Science. pp. 353-362. IEEE Computer Society (1989)

9. Clarke, E., Kroening, D., Lerda, F.: A tool for checking ANSI-C programs. In: Tools and Algorithms for the Construction and Analysis of Systems. LNCS, vol. 2988, pp. 168-176. Springer (2004)

10. Cook, B., Gulwani, S., Lev-Ami, T., Rybalchenko, A., Sagiv, M.: Proving conditional termination. In: CAV. LNCS, vol. 5123, pp. 328-340 (2008) 
11. David, C., Kesseli, P., Kroening, D., Lewis, M.: Danger invariants. In: Formal Methods. LNCS, vol. 9995, pp. 182-198. Springer (2016)

12. Flanagan, C., Leino, K.R.M., Lillibridge, M., Nelson, G., Saxe, J.B., Stata, R.: Extended static checking for java. In: Programming Language Design and Implementation. pp. 234-245. ACM (2002)

13. Godefroid, P.: Compositional dynamic test generation. In: Proceedings of the 34th Annual ACM SIGPLAN-SIGACT Symposium on Principles of Programming Languages. pp. 47-54. POPL '07, ACM, New York, NY, USA (2007)

14. Godefroid, P., Lahiri, S.K., Rubio-González, C.: Statically validating must summaries for incremental compositional dynamic test generation. In: Proceedings of the 18th International Conference on Static Analysis. pp. 112-128. SAS'11, Springer-Verlag, Berlin, Heidelberg (2011)

15. Gulwani, S., Srivastava, S., Venkatesan, R.: Program analysis as constraint solving. In: Programming Language Design and Implementation. pp. 281-292. ACM (2008)

16. Harman, M., Hierons, R.M.: An overview of program slicing. Software Focus 2(3), 85-92 (2001)

17. Heizmann, M., Hoenicke, J., Podelski, A.: Termination analysis by learning terminating programs. In: Computer-Aided Verification. vol. 8559, pp. 797-813 (2014)

18. Komuravelli, A., Bjørner, N., Gurfinkel, A., McMillan, K.L.: Compositional verification of procedural programs using horn clauses over integers and arrays. In: Formal Methods in Computer-Aided Design. pp. 89-96 (2015)

19. Leino, K.R.M.: Dafny: An automatic program verifier for functional correctness. In: Logic for Programming, Artificial Intelligence, and Reasoning. LNCS, vol. 6355, pp. 348-370. Springer (2010)

20. Miné, A.: Inferring sufficient conditions with backward polyhedral underapproximations. Electr. Notes Theor. Comput. Sci. 287, 89-100 (2012)

21. Namjoshi, K.S., Trefler, R.J.: On the completeness of compositional reasoning methods. ACM Trans. Comput. Log. 11(3) (2010)

22. Sankaranarayanan, S., Ivancic, F., Shlyakhter, I., Gupta, A.: Static analysis in disjunctive numerical domains. In: SAS. LNCS, vol. 4134, pp. 3-17 (2006)

23. Schrammel, P.: Challenges in decomposing encodings of verification problems. In: Horn Clauses for Verification and Synthesis. p. to appear. EPTCS (2016)

24. Schrammel, P., Kroening, D.: 2LS for Program Analysis - (Competition Contribution). In: TACAS. LNCS, vol. 9636, pp. 905-907 (2016)

25. Schrammel, P., Kroening, D., Brain, M., Martins, R., Teige, T., Bienmüller, T.: Successful use of incremental BMC in the automotive industry. In: Formal Methods for Industrial Critical Systems. LNCS, vol. 9128, pp. 62-77. Springer (2015)

26. Sery, O., Fedyukovich, G., Sharygina, N.: Interpolation-based function summaries in bounded model checking. In: HVC. LNCS, vol. 7261, pp. 160-175 (2011)

27. SPARK: (2014), http://www.spark-2014.org/ 